\title{
Population Pharmacokinetics of Unbound and Total Teicoplanin in Critically III Pediatric Patients
}

\author{
L. B. S. Aulin ${ }^{1} \cdot$ P. De Paepe ${ }^{2} \cdot$ E. Dhont ${ }^{3} \cdot$ A. de Jaeger ${ }^{3} \cdot J_{\text {. Vande Walle }}^{4} \cdot$ W. Vandenberghe ${ }^{3} \cdot$ B. C. McWhinney ${ }^{5}$. \\ J. P. J. Ungerer ${ }^{5,6}$. J. G. C. van Hasselt ${ }^{1}$ • P. A. J. G. De $\operatorname{Cock}^{2,3,7}$
}

Accepted: 16 September 2020

(c) Springer Nature Switzerland AG 2020

\begin{abstract}
Background and Objectives Teicoplanin is a highly protein-bound antibiotic, increasingly used to treat serious Gram-positive infections in critically ill children. Maturational and pathophysiological intensive care unit-related changes often lead to altered pharmacokinetics. In this study, the objectives were to develop a pediatric population-pharmacokinetic model of unbound and total teicoplanin concentrations, to investigate the impact of plasma albumin levels and renal function on teicoplanin pharmacokinetics, and to evaluate the efficacy of the current weight-based dosing regimen.

Methods An observational pharmacokinetic study was performed and blood samples were collected for quantification of unbound and total concentrations of teicoplanin after the first dose and in assumed steady-state conditions. A populationpharmacokinetic analysis was conducted using a standard sequential approach and Monte Carlo simulations were performed for a probability of target attainment analysis using previously published pharmacokinetic-pharmacodynamic targets.

Results A two-compartment model with allometric scaling of pharmacokinetic parameters and non-linear plasma protein binding best described the data. Neither the inclusion of albumin nor the renal function significantly improved the model and no other covariates were supported for inclusion in the final model. The probability of target attainment analysis showed that the standard dosing regimen does not satisfactory attain the majority of the proposed targets.

Conclusions We successfully characterized the pharmacokinetics of unbound and total teicoplanin in critically ill pediatric patients. The highly variable unbound fraction of teicoplanin could not be predicted using albumin levels, which may support the use of therapeutic drug monitoring of unbound concentrations. Poor target attainment was shown for the most commonly used dosing regimen, regardless of the pharmacokinetic-pharmacodynamic target evaluated.
\end{abstract}

Electronic supplementary material The online version of this article (https://doi.org/10.1007/s40262-020-00945-4) contains supplementary material, which is available to authorized users.

P. A. J. G. De Cock

pieter.decock@uzgent.be

1 Systems Biomedicine and Pharmacology, Leiden Academic Centre for Drug Research, Leiden University, Leiden, The Netherlands

2 Heymans Institute of Pharmacology, Ghent University, Ghent, Belgium

3 Department of Pediatric Intensive Care, Ghent University Hospital, Ghent, Belgium
4 Department of Pediatric Nephrology, Ghent University Hospital, Ghent, Belgium

5 Department of Chemical Pathology, Pathology Queensland, Brisbane, QLD, Australia

6 School of Biomedical Sciences, University of Queensland, Brisbane, QLD, Australia

7 Department of Pharmacy, Ghent University Hospital, De Pintelaan 185, 9000 Ghent, Belgium 


\section{Key Points}

Albumin was not found to be predictive of the unbound fraction of teicoplanin in the studied population and thus might lack clinical relevance for predicting unbound teicoplanin pharmacokinetics.

Estimated glomerular filtration rate based on serum creatinine or cystatin $\mathrm{C}$ was not predictive of teicoplanin clearance in the studied pediatric population.

An overall poor target attainment was observed, regardless of the pharmacokinetic-pharmacodynamic target used.

\section{Introduction}

Hospital-acquired infections caused by Gram-positive bacteria are associated with high mortality in critically ill pediatric patients. Gram-positive infections have historically been treated with vancomycin and this antibiotic is still extensively used. However, in pediatric patients, there is an increasing use of teicoplanin. This increase could be attributed to when compared with vancomycin, teicoplanin achieves equivalent efficacy while having a more favorable adverse-effect profile [1, 2]. The increasing use of teicoplanin in pediatric patients warrants further characterization of its patient group-specific pharmacokinetics to allow for treatment optimization.

The currently recommended dosing regimen of teicoplanin in pediatric patients consists of a loading phase of three $10-\mathrm{mg} / \mathrm{kg}$ intravenous doses given with a $12-\mathrm{h}$ interval, followed by a maintenance phase of $10-\mathrm{mg} / \mathrm{kg}$ intravenous dosing once daily [3]. Although therapeutic drug monitoring of teicoplanin in pediatric populations has been recommended [4], it is not commonly routinely performed. This is most likely due to the lack of an established PK-pharmacodynamic (PK-PD) target of teicoplanin [5]. Several conflicting PK-PD targets have been reported for teicoplanin (Table 1), but there is to date no consensus regarding which target to optimize treatment.

Critically ill pediatric patients may display highly variable pharmacokinetics. This variability can be attributed to changes in organ function due to maturation processes, pathophysiological changes, and drug-drug interactions [6]. Changes in plasma protein binding in critically ill patients represent another factor that may affect the pharmacokinetics of drugs [7]. As the unbound concentration of antibiotics represents the pharmacologically active driver of anti-bacterial drug action, consideration of the unbound fraction of antibiotics in critically ill patients is of major relevance [7]. Teicoplanin is a mixture of several isomorphic components, including five major compounds (A2-1 to 5) accounting for $95 \%$ of the total product, an hydrolysis product (A3-1), and

Table 1 Previously published pharmacokinetic-pharmacodynamic (PK-PD) and surrogate PK targets of teicoplanin

\begin{tabular}{|c|c|c|c|c|c|}
\hline $\begin{array}{l}\text { PK-PD or surrogate PK } \\
\text { target }\end{array}$ & Population $(n)$ & PD endpoint & Type of analysis & Type of study & References \\
\hline$C_{\text {min, day } 3} \geq 20 \mathrm{mg} / \mathrm{L}$ & Endocarditis (31) & Cure or fail & Fisher's test & Open & {$[36]$} \\
\hline$C_{\min }>20 \mathrm{mg} / \mathrm{L}$ & Septicemia (78) & Cure or fail & Logistic regression & Retrospective & {$[37]$} \\
\hline $\mathrm{AUC}_{\text {day } 3} \geq 750 \mathrm{mg} \times \mathrm{h} / \mathrm{L}$ & Patients with MRSA (24) & $\begin{array}{l}\text { Bacteriological eradica- } \\
\text { tion or persistence }\end{array}$ & Logistic regression & Retrospective & {$[38]$} \\
\hline $\mathrm{AUC}_{\text {day } 3} \geq 800 \mathrm{mg} \times \mathrm{h} / \mathrm{L}$ & Patients with MRSA (33) & $\begin{array}{l}\text { End of therapy eradica- } \\
\text { tion of MRSA }\end{array}$ & Logistic regression & Retrospective & [39] \\
\hline $\mathrm{AUC}_{\text {day } 1} / \mathrm{MIC} \geq 900$ & Patients with MRSA (42) & $\begin{array}{l}\text { Semi-quantitative bacte- } \\
\text { rial efficacy }\end{array}$ & Logistic regression & Retrospective & [40] \\
\hline $\mathrm{AUC}_{\text {day } 1} / \mathrm{MIC} \geq 610.4$ & $\begin{array}{l}\text { Neutropenic mice thigh } \\
\text { infection }(36)\end{array}$ & $2 \log 10$ bacterial kill & popPK-PD modeling & In vivo dose fractionation & [4] \\
\hline $\mathrm{AUC}_{\text {day } 1} / \mathrm{MIC} \geq 1500$ & $\begin{array}{l}\text { Neutropenic mice thigh } \\
\text { infection }(36)\end{array}$ & $\begin{array}{l}\text { Suppression of resistant } \\
\text { bacterial population at } \\
\text { end of therapy }\end{array}$ & popPK-PD modeling & In vivo dose fractionation & [4] \\
\hline$f \mathrm{AUC}_{\text {day } 5} / \mathrm{MIC} \geq 576$ & HFIM (30) & $2 \log 10$ bacterial kill & popPK-PD modeling & $\begin{array}{l}\text { In vitro dose fractiona- } \\
\text { tion }\end{array}$ & [4] \\
\hline$f \mathrm{AUC}_{\text {day } 5} / \mathrm{MIC} \geq 1326$ & HFIM (30) & $\begin{array}{l}\text { Suppression of resistant } \\
\text { bacterial population at } \\
\text { end of therapy }\end{array}$ & popPK-PD modeling & $\begin{array}{l}\text { In vitro dose fractiona- } \\
\text { tion }\end{array}$ & [4] \\
\hline
\end{tabular}

$A U C$ area under the curve, $C_{\min }$ minimum concentration, $M I C$ minimum inhibitory concentration, $H F I M$ hollow fiber infection model, $M R S A$ methicillin-resistant Staphylococcus aureus, pop population 
four minor (RS-1 to 4) compounds. All main compounds are extensively protein bound (total teicoplanin protein binding [>95\%] [8]), but show slightly variable affinity to albumin [9]. Because of this extensive protein binding, changes in plasma protein concentrations can influence teicoplanin efficacy [10].

Teicoplanin is exclusively renally excreted, primarily through glomerular filtration [11]. The glomerular filtration rate (GFR) is therefore considered a key determinant of teicoplanin clearance. The GFR in pediatric patients is subjected to maturation up to approximately 1 year of age [12]. Clearance of teicoplanin in pediatric patients can therefore be affected by maturational effects in the GFR. Pathophysiological effects on renal function may further affect the renal clearance of teicoplanin [13]. Several formulas to estimate the GFR in pediatric patients have been derived. Most of these approximations are based on either serum creatinine $\left(\mathrm{S}_{\mathrm{Cr}}\right)$, serum cystatin $\mathrm{C}$ (cysC), or a combination of these markers [14]. However, a consensus regarding the most predictive formula to reflect the GFR in children is still lacking.

The pharmacokinetics of total teicoplanin concentrations in pediatric populations has previously been described using two-compartment population-PK models [15-18]. Unbound teicoplanin pharmacokinetics has been described for an adult patient population with hematological malignancies [19]. However, characterization of unbound teicoplanin pharmacokinetics in the pediatric population is currently lacking.

The current study addresses the knowledge gap of unbound teicoplanin pharmacokinetics in the pediatric population. We aimed to (1) develop a population-PK model for unbound and total teicoplanin pharmacokinetics in pediatric critically ill patients, (2) investigate the predictive quality of patient-specific differences in albumin levels and GFR metrics on inter-individual variability (IIV) in PK parameters, and (3) evaluate target attainment of the current standard weight-based dosing regimen using several PK-PD targets.

\section{Methods}

\subsection{Study Design and Patients}

A prospective PK study (ClinicalTrials.gov number NCT02456974) was conducted at the pediatric intensive care unit of the Ghent University Hospital, Ghent, Belgium, between May 2012 and September 2017. Patients between the age of 1 month and 15 years were included upon admission to the pediatric intensive care unit if treatment with intravenous teicoplanin was clinically indicated. Patients were excluded if they lacked a catheter for blood sampling, if there was a documented hypersensitivity to aminoglycosides, or if they were on an extracorporeal circuit. Collected demographic and clinical variables included bodyweight
(BW), height, primary reason for admission, measures of organ function and patient severity of illness as described by the pediatric logistic organ dysfunction (PELOD) score, the pediatric risk of mortality (PRISM) II score, presence of mechanical ventilation, co-treatment with vasopressors, nephrotoxic medications, and highly plasma protein-bound drugs, presence of surgery, fluid resuscitation, albumin level, $\mathrm{S}_{\mathrm{Cr}}$, cysC, and C-reactive protein (CRP) level.

\subsection{Drug Dosing and Administration}

Teicoplanin (Targocid ${ }^{\circledR} 400 \mathrm{mg}$; Sanofi, Diegem, Belgium) was prescribed in a dose of $10 \mathrm{mg} / \mathrm{kg}$ BW every $12 \mathrm{~h}$ for three doses, thereafter every $24 \mathrm{~h}$. Teicoplanin was administered intravenously over 3-30 min using a calibrated syringe driver.

\subsection{Pharmacokinetic Sampling}

Serial blood samples were obtained from the first dose and/ or doses $>24 \mathrm{~h}$ after the start of treatment from an indwelling catheter other than the drug infusion line. The total number of samples collected (per individual patient) was limited by the predefined total maximum blood volume permitted for PK sampling (i.e., $2.4 \mathrm{~mL} / \mathrm{kg} \mathrm{BW}$ ). A typical sampling scheme included blood sampling just before dosing, a sample immediately after dosing and a flush, a distribution sample between 5 and 360 min after the start of infusion, a middose sample, and a trough sample just prior to the next dose. All samples were immediately transferred on to ice to the chemistry laboratory and centrifuged $(8 \mathrm{~min}$ at $1885 \mathrm{~g}$ ), after which the resulting plasma was frozen at $-80{ }^{\circ} \mathrm{C}$ before a bioanalytical analysis was performed.

\subsection{Bioanalytical Pharmacokinetic Assay}

Unbound and total plasma concentrations of teicoplanin (A2-1, A2-2, A2-3, A2-4, A2-5, A3-1) were quantified using a validated, reverse-phase, high-performance liquid chromatography method with ultraviolet detection. The lower limit of quantification (LLOQ) was $0.5 \mathrm{mg} / \mathrm{L}$ and the coefficient of variation $(\mathrm{CV})$ was $<10 \%$ at all levels.

\subsection{Clinical Chemistry Assays}

Serum cys $C$ was measured using the Gentian immunoassay (Gentian AS, Moss, Norway) on an AU480 Chemistry Analyser (Beckman Coulter, Inc., Brea, CA, USA) [intraassay $\mathrm{CV}<4 \%$; inter-assay $\mathrm{CV}<2.4 \%]$. Serum creatinine was quantified using an isotope dilution mass spectrometry, traceable Jaffe rate method on a UniCel DxC 800 Synchron Clinical System (Beckman Coulter, Inc.). Serum CRP and 
albumin were measured on the Cobas 8000 (c502/c701) analyzer (Roche Diagnostics, Mannheim, Germany).

\subsection{Processing of Data}

Pharmacokinetic samples below the LLOQ were excluded from the analysis, which has been shown to be appropriate for a population-PK analysis when the number of LLOQ samples does not exceed 5\% [20]. Missing time-varying covariates were imputed with linear interpolation, next observation carried backwards, or last observation carried forward, depending on when the missingness occurred. If a covariate was completely missing for an individual, the population median was used.

\subsection{Base Model Development}

We evaluated one-, two-, and three-compartment models, with zero-order intra-vascular administration and first-order elimination for unbound $\left(C_{\mathrm{u}}\right)$ and total $\left(C_{\mathrm{tot}}\right)$ concentrations of teicoplanin simultaneously. The models were parameterized in terms of $C_{\mathrm{u}}$. Linear and non-linear plasma proteinbinding models were evaluated to quantify the relationship between $C_{\mathrm{u}}$ and $C_{\text {tot }}$ (Eqs. 1, 2).

$C_{\mathrm{tot}}=\frac{C_{\mathrm{u}}}{f_{\mathrm{u}}}$

$C_{\mathrm{tot}}=\frac{B_{\max } \times C_{\mathrm{u}}}{K_{\mathrm{D}}+C_{\mathrm{u}}}+C_{\mathrm{u}}$

Here, $f_{\mathrm{u}}$ is the unbound fraction of teicoplanin, $k_{\mathrm{D}}$ is the dissociation constant $(\mathrm{mg} / \mathrm{L})$, and $B_{\max }$ is the maximum protein-binding capacity $(\mathrm{mg} / \mathrm{L})$.

Inter-individual variability, including off-diagonal elements, was tested on all estimated structural parameters as follows:

$P_{i}=P \times e^{\eta_{i}}$,

where $P_{i}$ is the individual parameter estimate for the $i$ th individual, $P$ is the population parameter estimate, and $\eta_{i}$ is assumed $N\left(0, \omega^{2}\right)$.

Residual unexplained variability was considered according to additive, proportional, or combined error models. Separate error models were implemented for the $C_{\text {tot }}$ and the $C_{\mathrm{u}}$, respectively. The selection of error model was based on a $-2 \log$-likelihood ( $-2 \mathrm{LL}$ ) and residual diagnostics.

\subsection{Covariate Model Development Strategy}

The covariate model development, i.e., the identification of predictors for IIV in PK parameters, was performed in a stepwise manner with a priori inclusion of BW on clearances and volumes of distribution. Bodyweight was normalized over the population median [21] and used with an allometric exponent of 1 and 0.75 for volumes of distribution and clearances [12, 22], respectively. We have chosen the approach of a priori size-based scaling based on BW because of (1) the large differences in BW and therefore the need to account for body size differences and (2) the limited number of patients included, which may result in inferior parameter precision of the covariate effect parameter.

Albumin-dependent binding was evaluated for both linear and non-linear implementations of the plasma proteinbinding model. Albumin dependency of the linear-binding model (Eq. 4) was implemented as a linear relationship with albumin plasma levels $\left(C_{\text {albumin }}\right)$. Tested non-linear albumindependent protein-binding models included two variations of a saturation model, that either included a linearly albumin-dependent maximum binding capacity $\left(B_{\text {max }}\right)($ Eq. 5 ) or mechanistically derived $B_{\max }$ (Eq. 6) [19].

$C_{\text {tot }}=\frac{C_{\mathrm{u}}}{f_{\mathrm{u}}} \times \frac{C_{\text {albumin }}}{C_{\text {albumin }_{\text {median }}}}$

$B_{\text {max }}=\frac{C_{\text {albumin }}}{C_{\text {albumin }_{\text {median }}}} \times \theta_{B_{\max }}$

$B_{\text {max }}=C_{\text {albumin }} \times 1.23 \times \frac{\mathrm{Mw}_{\text {teicoplanin }}}{\mathrm{Mw}_{\text {albumin }}} \times 1000$.

Here, $C_{\text {albumin }}$ is the albumin level $(\mathrm{g} / \mathrm{L}), k_{\mathrm{D}}$ is the dissociation constant $(\mathrm{mg} / \mathrm{L}), B_{\max }$ is the maximum protein-binding capacity $(\mathrm{mg} / \mathrm{L}), 1.23$ is the average number of teicoplaninbinding sites per albumin molecule in respect to the different isoforms [9, 19], and $\mathrm{Mw}_{\text {teicoplanin }}$ and $\mathrm{Mw}_{\text {albumin }}$ is the molecular weight $(\mathrm{g} / \mathrm{mol})$ of teicoplanin and albumin, respectively.

For other covariates, we applied a stepwise strategy including forward inclusion $(p<0.05 ; 1$ degree of freedom, $\Delta-2 \mathrm{LL}<-3.84)$ and backwards elimination $(p>0.001 ; 1$ degree of freedom, $\Delta-2 \mathrm{LL}>-10.83$ ), based on the loglikelihood ratio test. Additional inclusion criteria for covariate effects were good precision in the point estimate (relative standard error $<40 \%$ ) and sufficient reduction in IIV for the parameter of interest (reduction in IIV $\geq 2 \%$ units of the $\mathrm{CV}$ ). Only clinically relevant associations were tested: sex, age, serum CRP, PELOD score, PRISM II score, renal function metrics, concomitant use of nephrotoxic drugs, vasodilators, and inotropic drugs on clearance, and sex, PELOD score, PRISM II score, and serum CRP on volumes of distribution. The effect of using highly protein-bound co-medications was tested on relevant model parameters related to protein binding.

Dichotomous covariates were modeled according to the following equation: 
$P=\theta_{\text {pop }} \times\left(\theta_{x}\right)^{x_{\text {bin }}}$,

where $P$ is the covariate adjusted population parameter, $\theta_{\text {pop }}$ is the baseline parameter estimate for parameter $P, \theta_{x}$ is the covariate effect estimate on $P$, and $x_{\text {bin }}$ is the presence (1) or absence $(0)$ of the binary covariate.

The inclusion of continuous covariates, except for renal function metrics, was evaluated using a linear or an exponential relationship according to the following formulas:

$P=\theta_{\text {pop }} \times\left(1+\theta_{x} \times \frac{x}{x_{\text {median }}}\right)$,

$P=\theta_{\text {pop }} \times\left(\frac{x}{x_{\text {median }}}\right)^{\theta_{x}}$

where $x$ is the covariate value, $x_{\text {median }}$ is the population median of the covariate, and $\theta_{x}$ is the covariate effect estimate on $P$.

An extensive evaluation of the relationship between descriptors for renal function and clearance was performed. Evaluated renal function descriptors included measured renal function biomarker concentrations as well as biomarker-based GFR estimates (eGFR), calculated from three different formulas and one semi-physiological maturation function.

The implementation of the renal function-dependent clearance was defined by separating total clearance of teicoplanin $\left(\mathrm{CL}_{\text {tot }}\right)$ into a renal function-dependent $\left(\theta_{\mathrm{CL}_{R}}\right)$ and independent clearance $\left(\mathrm{CL}_{\mathrm{NR}}\right)$. The renal biomarkers $\mathrm{S}_{\mathrm{Cr}}$ and cys $\mathrm{C}$ were tested as covariates on clearance according to the formula below:

$\mathrm{CL}_{\text {tot }}=\mathrm{CL}_{\mathrm{NR}}+\theta_{\mathrm{CL}_{R}} \times \frac{\mathrm{RB}}{\mathrm{RB}_{\text {median }}}$,

where $\mathrm{RB}$ is the renal function biomarker, $\mathrm{CL}_{\mathrm{NR}}$ is the renal function independent clearance, and $\theta_{\mathrm{CL}_{R}}$ is the renal function-dependent clearance. Additionally, we assessed an agedependent normalization of cysC, implemented as described by De Cock et al. [23].

The investigation of GFR-dependent clearance was implemented according to Eq. 11, where the eGFRs used were calculated using a formula based on $\mathrm{S}_{\mathrm{Cr}}$ (Eq. 12), cysC (Eq. 13), or both in combination (Eq. 14). Additionally, the inclusion of an upper limit of eGFR of $220 \mathrm{~mL} / \mathrm{min} / 1.73 \mathrm{~m}^{2}$ was evaluated for all three eGFRs.

$\mathrm{CL}_{\text {tot }}=\mathrm{CL}_{\mathrm{NR}}+\theta_{\mathrm{CL}_{R}} \times \frac{\mathrm{eGFR}}{\mathrm{eGFR}_{\text {median }}}$,

where $e G F R$ is derived from one of the equations below and $\mathrm{eGFR}_{\text {median }}$ is the population median.
Schwartz formula [24] : eGFR $=0.423 \times\left(\frac{\mathrm{HT}}{\mathrm{S}_{\mathrm{Cr}}}\right)^{0.79}$

Pottel formula [25] $:$ eGFR $=\frac{107.3 \times \text { cysC }}{0.82}$

Chehade formula [26] : eGFR

$$
\begin{aligned}
& =\frac{0.42 \times \mathrm{HT}}{\mathrm{S}_{\mathrm{Cr}}}-\left(\frac{0.0004 \times \mathrm{HT}}{\mathrm{S}_{\mathrm{Cr}}}\right)^{2} \\
& -14.5 \times \text { cysC }-0.69 \times \text { age }+Q
\end{aligned}
$$

where eGFR is the estimated glomerular filtration rate $(\mathrm{mL} /$ $\left.\mathrm{min} / 1.73 \mathrm{~m}^{2}\right)$, HT is height $(\mathrm{cm}), \mathrm{S}_{\mathrm{Cr}}$ is serum creatinine $(\mathrm{mg} / \mathrm{dL})$, cysC is serum cystatin $\mathrm{C}(\mathrm{mg} / \mathrm{L})$, and $Q$ is a sexspecific constant, which is 18.25 and 21.88 for female and male individuals, respectively.

Additionally, the implementation of a semi-physiological maturation function of GFR-mediated clearance was investigated as an alternative to the GFR formulas. The semi-physiological maturation function was implemented according to the following formula (Eq. 15):

$\mathrm{CL}_{\mathrm{tot}}=\mathrm{CL}_{\mathrm{NR}}+\theta_{\mathrm{CL}, \mathrm{GFR}} \times\left(\frac{\mathrm{BW}}{4000}\right)^{\mathrm{BDE}} ; \quad \mathrm{BDE}=2.23 \times \mathrm{BW}^{-0.065}$,

where $\mathrm{CL}_{\mathrm{NR}}$ is the GFR independent clearance, $\theta_{\mathrm{CL}, \mathrm{GFR}}$ is the population GFR-dependent clearance parameter, BW is in grams, and BDE is the BW-dependent exponent derived from the model reported by De Cock et al. [27].

\subsection{Model Evaluation}

The structural model was selected based on a combination of the likelihood-ratio test $(p<0.05)$, relative standard error of fixed-effect parameters $<40 \%$, and evaluation of graphical diagnostics, e.g., standard goodness-of-fit plots and prediction-corrected visual predictive check [28].

\subsection{Probability of Target Attainment Analysis}

Monte Carlo simulations were performed using the final model to generate five simulated patient populations, each representing a BW of $5,10,15,25$, and $50 \mathrm{~kg}(n=1000$ per weight group), respectively. The standard weight-based pediatric dosing regimen was used for all five populations, with each dose administered as a 5-min intravenous infusion. The probability of target attainment (PTA) was calculated for each PK-PD target reported in Table 1 and for each simulated population separately. Three different minimum inhibitory concentrations (MICs), between 0.25 and $1 \mathrm{mg} / \mathrm{L}$, were evaluated. The area under the curve (AUC) for days 1 , 
3 , and 5 was calculated as the cumulated exposure for $0-24$, 48-72, and 144-168 $\mathrm{h}$ after the first dose, respectively.

\subsection{Software}

The development of the population-PK model was performed using a non-linear effects modeling approach implemented in the NONMEM software (version 7.4.0). Subroutines for general non-linear models were used together with the first-order conditional estimation method with interaction throughout the analysis. The Monte Carlo simulations used for the PTA analysis were also performed in NONMEM. Perl-speaks-NONMEM (version 4.8.1) was used for the generation of simulation-based diagnostics, and R (3.5.0) was used for data processing and visualization.

\section{Results}

\subsection{Clinical Study}

A total of 42 pediatric patients were enrolled in the study. The demographics and clinical characteristics of the study population are summarized in Table 2. A total of $250 \mathrm{PK}$

Table 2 Demographics and clinical characteristics of pediatric patients included in the study $(n=42)$

\begin{tabular}{lll}
\hline Demographics & Unit & Median (range) \\
\hline Age & Years & $1.4(0.17-15.6)$ \\
Female & $\%$ & $40.5 \%$ \\
Weight & $\mathrm{kg}$ & $9.2(3.74-56)$ \\
Height & $\mathrm{cm}$ & $80.50(19-175)$ \\
PRISM II score & & $8(0-27)$ \\
PELOD score & & $1(0-22)$ \\
Ventilated & $\%$ & 52.3 \\
Volume resuscitation & $\%$ & 9.5 \\
Co-medications & & \\
Nephrotoxic & $\%$ & 28.6 \\
High protein binding & $\%$ & 97.6 \\
Vasopressor & $\%$ & 33.3 \\
Biomarkers & & \\
Serum CRP & & $29.7(0.10-224)$ \\
Serum albumin & $\mathrm{g} / \mathrm{L}$ & $30(18-46)$ \\
Serum creatinine & $\mathrm{mg} / \mathrm{dL}$ & $0.24(0.01-1.00)$ \\
Serum cystatin C & $\mathrm{mg} / \mathrm{L}$ & $0.81(0.28-1.99)$ \\
Estimated GFR & & $117.20(17.32-390.15)$ \\
Schwartz & $\mathrm{mL} / \mathrm{min} / 1.73 \mathrm{~m}^{2}$ & $104.68(36.64-260.40)$ \\
Pottel & $\mathrm{mL} / \mathrm{min} / 1.73 \mathrm{~m}^{2}$ & $161.73(21.64-715.24)$ \\
Chehade & $\mathrm{mL} / \mathrm{min} / 1.73 \mathrm{~m}^{2}$ & \\
\hline CRP C-reactie & &
\end{tabular}

$C R P$ C-reactive protein, GFR glomerular filtration rate, $P E L O D$ pediatric logistic organ dysfunction, PRISM pediatric risk of mortality plasma samples were collected, for which both total and unbound teicoplanin concentrations were determined. The median number of samples was five per patient. Two measurements of unbound teicoplanin $(0.8 \%)$ were below the LLOQ and were therefore excluded from the analysis. The missingness of time-varying covariates were $4.0 \%, 4.4 \%$, and $5.6 \%$ for albumin, cysC, and $\mathrm{S}_{\mathrm{Cr}}$, respectively.

\subsection{Population-Pharmacokinetic Model}

The PK data of teicoplanin were best described using a two-compartment model with albumin independent nonlinear protein-binding kinetics (Eq. 3) and first-order elimination (Figs. 1, 2, 3). The population-PK parameter estimates of the final model are provided in Table 3. The underlying ordinary differential equations describing the change of unbound teicoplanin over time in the central and peripheral compartments are stated below:

$\frac{\mathrm{d} A_{\mathrm{c}}}{\mathrm{d} t}=-\frac{C L}{V_{\mathrm{c}}} \times A_{\mathrm{c}}-\frac{Q}{V_{\mathrm{c}}} \times A_{\mathrm{c}}+\frac{Q}{V_{\mathrm{p}}} \times A_{\mathrm{p}}$,

$\frac{\mathrm{d} A_{\mathrm{p}}}{\mathrm{d} t}=\frac{Q}{V_{\mathrm{c}}} \times A_{\mathrm{c}}-\frac{Q}{V_{\mathrm{p}}} \times A_{\mathrm{p}}$,

where $A_{\mathrm{c}}$ and $A_{\mathrm{p}}$ is the unbound amount of teicoplanin in the central and peripheral compartments, respectively, $\mathrm{CL}$ is the clearance, $Q$ is the inter-compartmental clearance, and $V_{\mathrm{c}}$ and $V_{\mathrm{p}}$ is the central and peripheral volumes of distribution, respectively.

Inter-individual variability was estimated for CL (CV $34.4 \%), V_{\mathrm{c}}(\mathrm{CV} 56.3 \%), V_{\mathrm{p}}(\mathrm{CV} 36.3 \%), Q$ (CV 46.4\%), and $B_{\max }(\mathrm{CV} 36.3 \%)$. Off-diagonal elements were

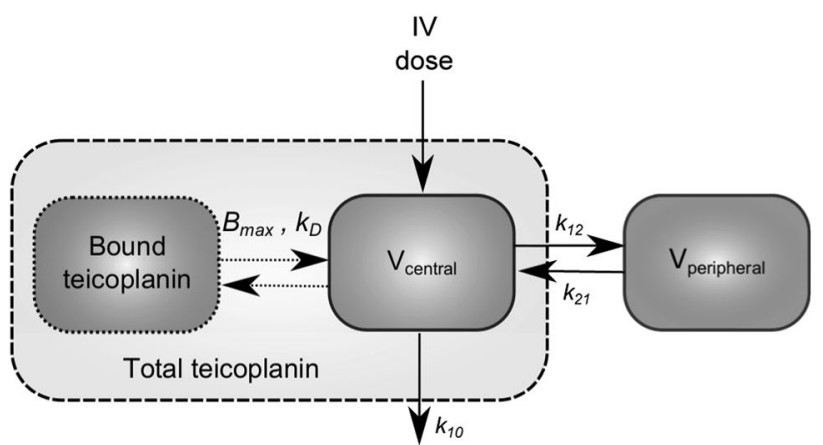

Fig. 1 Schematic of the developed two-compartment pharmacokinetic model with non-linear protein binding. $\mathrm{CL} / V_{\mathrm{c}}$ represents the elimination rate constant, $Q / V_{\mathrm{c}}$ and $Q / V_{\mathrm{p}}$ are distribution rate constants, $k_{\mathrm{D}}$ is the dissociation constant, and $B_{\max }$ is maximum protein binding. $I V$ intravenous 

ual predictive check of total (left panel) and unbound concentrations (right panel) of teicoplanin. Solid lines represent the observed 5th, 50th, and 95th percentiles of the observed data. The shaded areas represent $95 \%$ confidence intervals around the simulated percentiles
Fig. 2 Prediction-corrected vis-
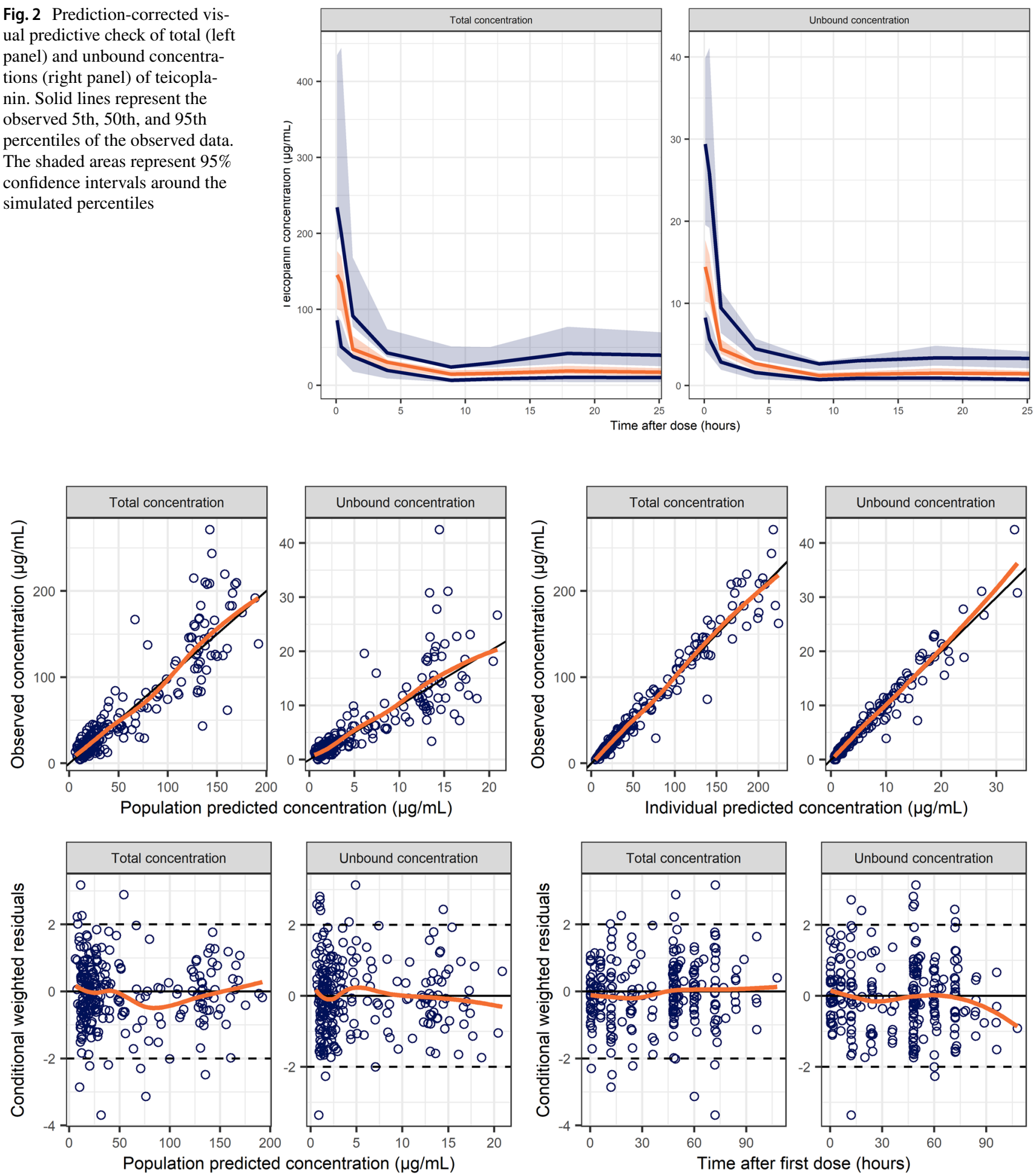

Fig. 3 Goodness-of-fit diagnostic plots for of unbound and total concentrations of teicoplanin. Plots showing observed concentrations vs predicted concentrations include a line of unity (black solid line) and a Loess smoother (orange solid line). Plots showing conditional weighted residuals include dashed lines indicating \pm 2 standard deviations 
Table 3 Parameter estimates for unbound teicoplanin for the final model

\begin{tabular}{lllll}
\hline Description & Parameter & Unit & Point estimate & RSE (\%) \\
\hline Structural model & & & & \\
Clearance & $\mathrm{CL}$ & $\mathrm{L} / \mathrm{h}$ & 1.95 & 5.8 \\
Central volume & $V_{\mathrm{c}}$ & $\mathrm{L}$ & 6.37 & 8.7 \\
Peripheral volume & $V_{\mathrm{p}}$ & $\mathrm{L}$ & 33.5 & 7 \\
Inter-compartmental clearance & $Q$ & $\mathrm{~L} / \mathrm{h}$ & 6.89 & 8.8 \\
Maximal binding capacity & $\theta_{B_{\max }}$ & $\mathrm{mg} / \mathrm{L}$ & 697 & 17.5 \\
Dissociation constant & $k_{\mathrm{D}}$ & $\mathrm{mg} / \mathrm{L}$ & 64.2 & 20.7 \\
Inter-individual variability & & & & 11 \\
CL & $\omega_{\mathrm{CL}}$ & $\mathrm{CV} \%$ & 34.4 & 17 \\
$V_{\mathrm{c}}$ & $\omega_{V_{\mathrm{c}}}$ & $\mathrm{CV} \%$ & 56.3 & 15 \\
$V_{\mathrm{p}}$ & $\omega_{V \mathrm{p}}$ & $\mathrm{CV} \%$ & 36.3 & 13 \\
$Q$ & $\omega_{Q}$ & $\mathrm{CV} \%$ & 46.4 & 14 \\
$B_{\text {max }}$ & $\omega_{B_{\max }}$ & $\mathrm{CV} \%$ & 36.3 & 18.5 \\
Residual variability & & $\mathrm{CV} \%$ & 2.99 & 19.9 \\
$\begin{array}{l}\text { Proportional error on total teicoplanin } \\
\quad \text { concentrations }\end{array}$ & $\sigma_{\mathrm{tot}}$ & & & \\
$\begin{array}{l}\text { Proportional error on unbound teico- } \\
\text { planin concentrations }\end{array}$ & $\sigma_{\mathrm{ub}}$ & $\mathrm{CV} \%$ & 4.15 & \\
\hline
\end{tabular}

$C V$ coefficient of variation, $R S E$ relative standard error estimated between CL, $V_{\mathrm{c}}, V_{\mathrm{p}}$, and $Q$ (covariance range 20.8-58.5\%).

Non-linear protein binding was found to significantly improve the description of the data compared with a linear implementation $(\Delta-2 \mathrm{LL}=-18.7)$. Introducing an empirical or mechanistic albumin-dependent binding did not improve the model.

\subsection{Covariate Analysis}

During the forward inclusion step, four statistically covariate relationships were identified; sex and $V_{c},(\Delta-2 \mathrm{LL}$ - 8.40), eGFR calculated using the Chechade formula (Eq. 14) and capped at $220 \mathrm{~mL} / \mathrm{min} / 1.73 \mathrm{~m}^{2}$, and CL $(\Delta$ $-2 \mathrm{LL}-5.24)$, inotropic co-medication and CL $(\Delta-2 \mathrm{LL}$ - 8.177), and CRP level and CL ( $\Delta-2 \mathrm{LL}-6.80)$. None of these relationships did however explain sufficient IIV to be considered clinically relevant ( $>2 \%$ units of $\mathrm{CV}$ ), thus the final model included no other covariate relationships than the a priori included relationships between $\mathrm{BW}$ and $V_{\mathrm{c}}, V_{\mathrm{p}}, \mathrm{CL}$, and $Q$.

\subsection{Probability of Target Attainment Analysis}

The PTA analysis showed that the standard dosing regimen did not achieve a satisfactory probability of success (PTA $>80 \%$ ) for the majority of PK-PD targets evaluated (Fig. 4). Higher weight was associated with greater
PTA for all targets. The PK-PD targets based on unbound teicoplanin concentration were associated with PTAs of below 5\%. The PK surrogate target most commonly used in the clinic, steady-state plasma trough concentration $\left(C_{\text {min }}\right.$,ay3 $)$, did not achieve satisfactory PTA for any population and was the target associated with the largest between-population difference (10.1 vs $52.4 \%$ ).

\section{Discussion}

To the best of our knowledge, this is the first population-PK model describing the pharmacokinetics and plasma protein binding of teicoplanin in a critically ill pediatric population. The developed model was based on rich data from a relevant cohort of patients after the start of teicoplanin treatment and in assumed steady-state conditions. The model provides novel insights into the study population-specific pharmacokinetics of this antibiotic. Thus, it can be used to guide further treatment optimization of teicoplanin treatment in critically ill children.

Teicoplanin is a highly protein-bound drug and is mainly bound to plasma albumin, of which concentrations may greatly vary within and between critically ill patients. In our patient population, we observed such variability $\left(C_{\text {albumin }}\right.$ : median $30.0 \mathrm{mg} / \mathrm{L}$, range $18-46 \mathrm{mg} / \mathrm{L})$. It has been shown that albumin levels significantly affect the unbound teicoplanin concentrations in adult patients [10, 29]. However, in our study, we could not identify such a relationship. As 


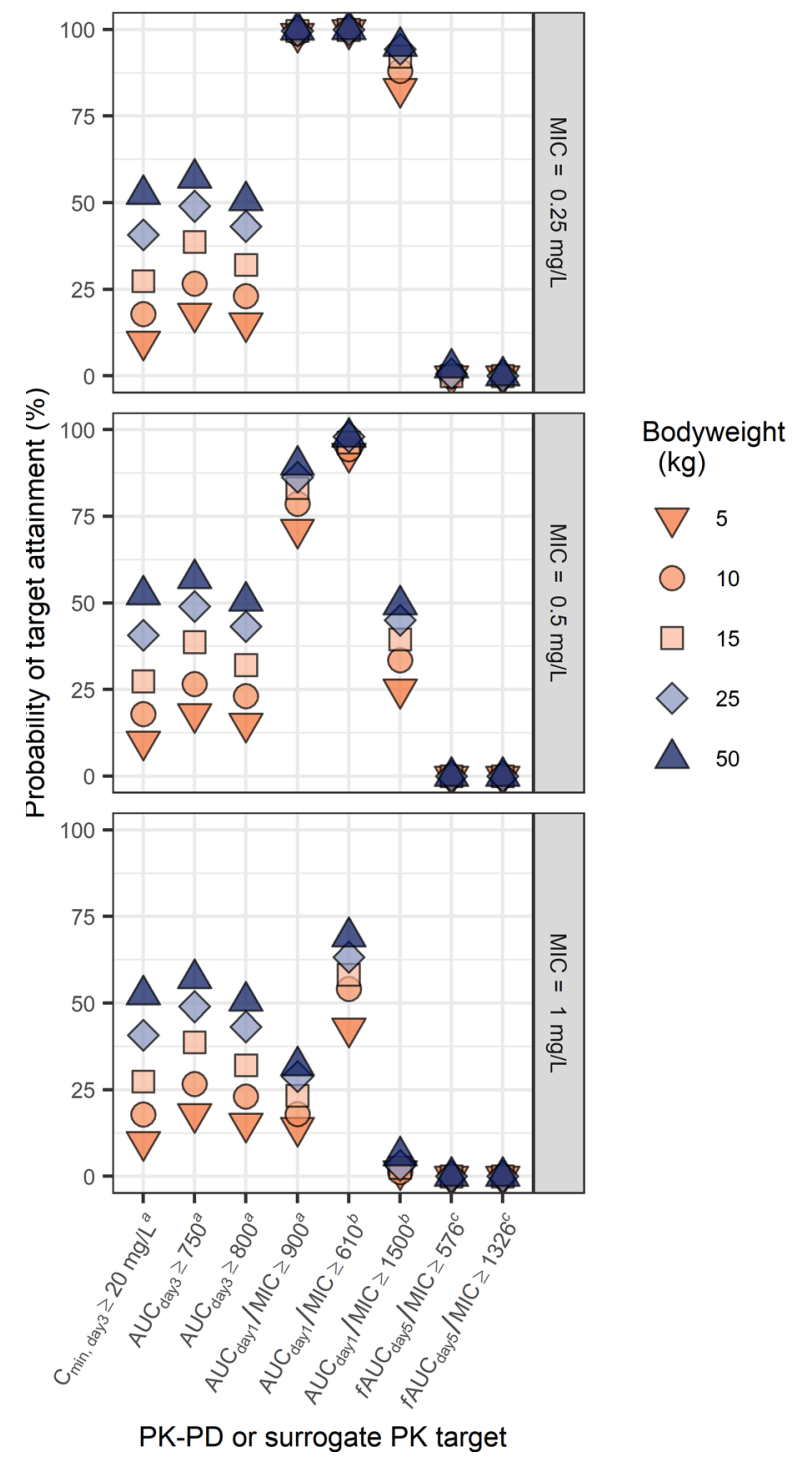

Fig. 4 Probability of target attainment (PTA) of eight different pharmacokinetic-pharmacodynamic (PK-PD) targets for five simulated pediatric patient populations $(n=1000)$ with different specific bodyweights given the standard pediatric teicoplanin dosing regimen and a minimum inhibitory concentration (MIC) of $0.25,0.5$, and $1 \mathrm{mg} / \mathrm{L}$. ${ }^{a}$ Clinically derived PK-PD and surrogate PK targets. ${ }^{\text {b }} \mathrm{PK}-\mathrm{PD}$ targets derived from in vivo dose fractionation studies in mice. ${ }^{\mathrm{C}} \mathrm{PK}-\mathrm{PD}$ targets derived from in vitro experiments based on unbound teicoplanin concentrations. Details on PK-PD targets are summarized in Table 1. $A U C$ area under the curve, $C_{\min }$ minimum concentration

we observed a high non-predictable variability in unbound fractions $\left(f_{\mathrm{u}}\right.$ : median 0.083 , range $\left.0.036-0.28\right)$, these observations support the measurement of unbound teicoplanin concentrations for optimization of treatment.

Teicoplanin is a renally cleared drug and its clearance is known to be decreased in the case of impaired renal function [30]. Renal function biomarkers, such as $\mathrm{S}_{\mathrm{Cr}}$ and cysC, are often used to obtain estimates of GFR as a measure of renal function. However, the reliability of these biomarkers in critically ill patients, especially pediatric, has been questioned [13]. Fluctuating $\mathrm{S}_{\mathrm{CR}}$ levels can be caused by many different factors unrelated to renal function, such as age, sex, muscle mass, physical activity, and nutrition [14, 31], making it a poor marker for renal function in critically ill pediatric patients. Cystatin $\mathrm{C}$ production, although less affected than $\mathrm{S}_{\mathrm{Cr}}$ by changes in muscle mass, can to some extent be altered by disease states often found in pediatric intensive care unit patients [32]. Additionally, rapid changes in renal function can occur in critically ill patients, due to e.g., sepsis. However, changes in serum concentrations of renal function biomarkers are often delayed [33], limiting their use in critically ill patients to assess GFR. A recently published study did however find eGFR using the Swartz $\mathrm{S}_{\mathrm{cr}}$-based formula to be predictive of teicoplanin clearance in a neonatal patient population [18]. This predictive ability of eGFR was also found in a study conducted in a pediatric population with hematological malignancies [16]. Both of these two study populations had higher $\mathrm{S}_{\mathrm{cr}}$ compared with our study population. The population included in our analysis had an overall elevated eGFR (Table 2), suggesting augmented clearance (Fig. S1 of the Electronic Supplementary Material). We were unable to establish a significant relationship between eGFR and teicoplanin clearance, thus strengthening the evidence that $\mathrm{S}_{\mathrm{Cr}^{-}}$and cysC-derived eGFRs are poor predictors of renal function in critically ill pediatric patients. Another potential explanation includes a too narrow variation in renal biomarkers to identify a statistically significant correlation, as no patients with renal failure were included. Additionally, including BW a priori could mask potential eGFR effects on clearance as these are widely known to be correlated. Although we could not identify a significant relationship between eGFR and clearance in our study, it does not disprove the existence of such a relationship. However, using BW as the sole predictor of teicoplanin clearance is clinically advantageous because of its availability.

In this study, we compared target attainment using evidence-based PK-PD targets (Table 1). Our analysis showed that the current weight-based standard pediatric dosing regimen results in significant under-dosing regardless of the PK-PD target used for infections caused by pathogens in the upper MIC range. A trend of higher attainment rates in children with higher BW compared with children with lower BW was observed (Fig. 4). These observations are in line with previously published research in children aged older than 1 month [16]. It is important to take into account potential differences in a bio-analysis method. Immunoassays suffer from non-specific interferences because the used antibodies can also interact with compounds other than the main teicoplanin isomorphic compounds $[34,35]$. 
Large differences in target attainment rates were noted, depending on the PK-PD or surrogate PK target used. Dose fractionation studies are often performed to characterize the driver of the antibacterial effect and derive target values. A well-designed dose fractionation study of teicoplanin has been published, deriving an in vivo $\mathrm{PK}-\mathrm{PD}$ target for both efficacy $\left(\mathrm{AUC}_{\text {day } 1} / \mathrm{MIC} 610\right)$ and suppression of antibiotic resistance with methicillin-resistant Staphylococcus aureus ( $\left.\mathrm{AUC}_{\mathrm{day} 1} / \mathrm{MIC} 1500\right)$, based on total drug concentrations [4]. Using the current standard dosing regimen, these target are attained in critically ill pediatric patients for pathogens with MICs up to $0.5 \mathrm{mg} / \mathrm{L}$ (Fig. 4). This is in accordance with what was suggested by Ramos-Martín et al. [4]. In clinical practice, total trough concentration (minimum concentration $\left[C_{\min }\right]$ ) is used as a surrogate PK parameter for a target total AUC exposure. The most commonly used clinical target is $C_{\min }$ above $20 \mathrm{mg} / \mathrm{L}$. In our patient population, an overall $29.4 \%$ of patients achieved this PK target.

The unbound fraction of teicoplanin is highly variable in our patient population and only the unbound concentration exerts a pharmacological effect, a PK-PD target based on unbound concentrations would be most suitable for target attainment evaluation. The only currently available PK-PD targets based on unbound teicoplanin concentrations are based on data from an in vitro hollow fiber infection model with methicillin-resistant $S$. aureus [4]. Although the hollow fiber infection model is a powerful in vitro model with the capacity of simulating in vivo pharmacokinetics, it suffers from some disadvantages compared with in vivo models. One of these disadvantages is the lack of effect of the immune response, thus underestimating the in vivo efficacy. Additionally, hollow fiber infection model experiments are usually conducted in rich media, an environment that is highly different in regard to nutrient availability compared with in vivo. This environment greatly favors bacterial growth, leading to super-physiological growth rates. Because of the extensive binding of teicoplanin, the current dosing regimen fails to attain these $f \mathrm{AUC} / \mathrm{MIC}_{\mathrm{day} 5}$ targets for efficacy (576) and suppression of antimicrobial resistance (1326) for pathogens with MICs $\geq 0.25 \mathrm{mg} / \mathrm{L}$.

\section{Conclusions}

We successfully characterized the pharmacokinetics of unbound and total teicoplanin in critically ill pediatric patients. We showed that the highly variable unbound fraction of teicoplanin could not be predicted using albumin levels. Because of the relatively high inter-individual variation in unbound teicoplanin concentrations that cannot be predicted with covariates, routine therapeutic drug monitoring of unbound concentrations may be recommended in the clinic to guide treatment optimization in critically ill pediatric patients. A poor target attainment was obtained with the most commonly used dosing regimen, regardless of the PK-PD target evaluated. To properly optimize treatment based on unbound concentrations, more focused in vivo and clinical research on PK-PD targets using unbound concentrations for the efficacy and suppression of antimicrobial resistance is warranted.

Acknowledgements We thank Sarah Desmet, Margot Wollaert, and SAFEPEDRUG study nurses (Daphne, Anca, Fien) for their invaluable help with blood sampling and/or data management.

\section{Declarations}

Funding This work was supported by the Clinical Research Fund Ghent University Hospital, Ghent Belgium (grant number WR/1294/ APO/001 to Pieter A. J. G. De Cock) and the Agency for Innovation by Science and Technology, Flanders, Belgium (SAFEPEDRUG project; grant number IWT/SBO/130033).

Conflict of Interest Not applicable.

Ethics Approval The study was conducted in accordance with the guidelines of the Declaration of Helsinki, was approved by the institutional ethics committee (EC/2012/172), and was registered at ClinicalTrials.gov (NCT02456974).

Consent to Participate Written informed consent was obtained from the parents or legal representatives, and assent was obtained from patients aged older than 12 years.

Consent for Publication Not applicable.

Availability of Data and Material Not applicable.

Code Availability Not applicable.

Authors' Contributions Not applicable.

\section{References}

1. Sidi V, Roilides E, Bibashi E, Gompakis N, Tsakiri A, Koliouskas D. Comparison of efficacy and safety of teicoplanin and vancomycin in children with antineoplastic therapy-associated febrile neutropenia and Gram-positive bacteremia. J Chemother. 2000;12(4):326-31.

2. Cavalcanti AB, Goncalves AR, Almeida CS, Bugano DDG, Silva E. Teicoplanin versus vancomycin for proven or suspected infection. Cochrane Database Syst Rev. 2010;6:6-8.

3. Sanofi. Targocid 400mg: summary of product characteristics. 2017.

4. Ramos-Martín V, Johnson A, McEntee L, Farrington N, Padmore $\mathrm{K}$, Cojutti $\mathrm{P}$, et al. Pharmacodynamics of teicoplanin against MRSA. J Antimicrob Chemother. 2017;72(12):3382-9.

5. Boast A, Gwee A. Teicoplanin: should we be doing routine therapeutic drug monitoring in children? Pediatr Infect Dis J. 2017;36(11):1110. 
6. Lu H, Rosenbaum S. Developmental pharmacokinetics in pediatric populations. J Pediatr Pharmacol Ther. 2014;19(4):262-76.

7. Ulldemolins M, Roberts JA, Rello J, Paterson DL, Lipman J. The effects of hypoalbuminaemia on optimizing antibacterial dosing in critically ill patients. Clin Pharmacokinet. 2011;50(2):99-110.

8. Dykhuizen RS, Harvey G, Stephenson N, Nathwani D, Gould IM. Protein binding and serum bactericidal activities of vancomycin and teicoplanin. Antimicrob Agents Chemother. 1995;39(8):1842-7.

9. Assandri A, Bernareggi A. Binding of teicoplanin to human serum albumin. Eur J Clin Pharmacol. 1987;33:191-5.

10. Yano R, Nakamura T, Tsukamoto H, Igarashi T, Goto N, Wakiya $\mathrm{Y}$, et al. Variability in teicoplanin protein binding and its prediction using serum albumin concentrations. Ther Drug Monit. 2007;29(4):399-403.

11. Wilson AP. Clinical pharmacokinetics of teicoplanin. Clin Pharmacokinet. 2000;39(3):167-83.

12. Rhodin MM, Anderson BJ, Peters AM, Coulthard MG, Wilkins B, Cole M, et al. Human renal function maturation: a quantitative description using weight and postmenstrual age. Pediatr Nephrol. 2009;24(1):67-766.

13. Dhont E, Van Der Heggen T, De Jaeger A, Vande Walle J, De Paepe P, De Cock PA. Augmented renal clearance in pediatric intensive care: are we undertreating our sickest patients? Pediatr Nephrol. 2020;35(1):25-39.

14. den Bakker E, Gemke RJBJ, Bökenkamp A. Endogenous markers for kidney function in children: a review. Crit Rev Clin Lab Sci. 2018;55(3):163-83.

15. Ramos-Martín V, Paulus S, Siner S, Scott E, Padmore K, Newland $\mathrm{P}$, et al. Population pharmacokinetics of teicoplanin in children. Antimicrob Agents Chemother. 2014;58(11):6920-7.

16. Zhao W, Zhang D, Storme T, Baruchel A, Declèves X, JacqzAigrain E. Population pharmacokinetics and dosing optimization of teicoplanin in children with malignant haematological disease. Br J Clin Pharmacol. 2015;80(5):1197-207.

17. Lukas JC, Karikas G, Gazouli M, Kalabalikis P, Hatzis T, Macheras P. Pharmacokinetics of teicoplanin in an ICU population of children and infants. Pharm Res. 2004;21(11):2064-71.

18. Kontou A, Sarafidis K, Begou O, Gika HG, Tsiligiannis A, Ogungbenro K, et al. Population pharmacokinetics of teicoplanin in preterm and term neonates: is it time for a new dosing regimen? Antimicrob Agents Chemother. 2020;64(4):e01971-e2019.

19. Byrne CJ, Parton T, McWhinney B, Fennell JP, O'Byrne P, Deasy E, et al. Population pharmacokinetics of total and unbound teicoplanin concentrations and dosing simulations in patients with haematological malignancy. J Antimicrob Chemother. 2018;74(4):995-1003

20. Xu XS, Dunne A, Kimko H, Nandy P, Vermeulen A. Impact of low percentage of data below the quantification limit on parameter estimates of pharmacokinetic models. J Pharmacokinet Pharmacodyn. 2011;38(4):423-32.

21. Goulooze SC, Völler S, Välitalo PAJ, Calvier EAM, Aarons L, Krekels EHJ, et al. The influence of normalization weight in population pharmacokinetic covariate models. Clin Pharmacokinet. 2019;58(1):131-8.

22. Germovsek E, Barker CIS, Sharland M, Standing JF. Scaling clearance in paediatric pharmacokinetics: all models are wrong, which are useful? Br J Clin Pharmacol. 2017;83(4):777-90.

23. De Cock PAJG, van Dijkman SC, de Jaeger A, Willems J, Carlier M, Verstraete AG, et al. Dose optimization of piperacillin/ tazobactam in critically ill children. J Antimicrob Chemother. 2017;72(7):2002-111.

24. Schwartz GJ, Schneider MF, Maier PS, Moxey-Mims M, Dharnidharka VR, Warady B, et al. Improved equations estimating GFR in children with chronic kidney disease using an immunonephelometric determination of cystatin C. Kidney Int. 2012;82(4):445-53.

25. Pottel H, Delanaye P, Schaeffner E, Dubourg L, Eriksen BO, Melsom T, et al. Estimating glomerular filtration rate for the full age spectrum from serum creatinine and cystatin C. Nephrol Dial Transplant. 2017;32(3):497-507.

26. Chehade H, Cachat F, Jannot AS, Meyrat BJ, Mosig D, Bardy D, et al. New combined serum creatinine and cystatin $\mathrm{C}$ quadratic formula for GFR assessment in children. Clin J Am Soc Nephrol. 2014;9(1):54-63.

27. De Cock RFW, Allegaert K, Brussee JM, Sherwin CMT, Mulla H, De Hoog M, et al. Simultaneous pharmacokinetic modeling of gentamicin, tobramycin and vancomycin clearance from neonates to adults: towards a semi-physiological function for maturation in glomerular filtration. Pharm Res. 2014;31(10):2643-54.

28. Nguyen THT, Mouksassi M, Holford N, Freedman I, Hooker AC, John J, et al. Model evaluation of continuous data pharmacometric models: metrics and graphics. CPT Pharmacomet Syst Pharmacol. 2017;6(2):87-109.

29. Brink AJ, Richards GA, Lautenbach EEG, Rapeport N, Schillack V, Van Niekerk L, et al. Albumin concentration significantly impacts on free teicoplanin plasma concentrations in non-critically ill patients with chronic bone sepsis. Int J Antimicrob Agents. 2015;45(6):647-51.

30. Falcoz C, Ferry N, Pozet N, Cuisinaud G, Zech PY, Sassard J. Pharmacokinetics of teicoplanin in renal failure. Antimicrob Agents Chemother. 1987;31(8):1255-62.

31. Hari P, Bagga A, Mahajan P, Lakshmy R. Effect of malnutrition on serum creatinine and cystatin $\mathrm{C}$ levels. Pediatr Nephrol. 2007;22(10):1757-61.

32. Andersen TB, Eskild-Jensen A, Frøkiær J, Brøchner-Mortensen J. Measuring glomerular filtration rate in children; can cystatin $\mathrm{C}$ replace established methods? A review. Pediatr Nephrol. 2009;24(5):929-41.

33. Soni SS, Ronco C, Katz N, Cruz DN. Early diagnosis of acute kidney injury: the promise of novel biomarkers. Blood Purif. 2009;28(3):165-74.

34. Davani S, Brard M, Royer B, Kantelip JP, Muret P. Comparison of fluorescence polarization immunoassay and high-performance liquid chromatography methods for assay of teicoplanin: can correlation be improved? Pathol Biol (Paris). 2004;52(10):584-8.

35. Mueller DM, Von Eckardstein A, Saleh L. Quantification of teicoplanin in plasma by LC-MS with online sample cleanup and comparison with QMS ${ }^{\circledR}$ assay. Clin Chem Lab Med. 2014;52(6):879-87.

36. Wilson AP, Grüneberg RN, Neu H. A critical review of the dosage of teicoplanin in Europe and the USA. Int J Antimicrob Agents. 1994;4(Suppl 1):1-30.

37. Harding I, MacGowan AP, White LO, Darley ESR, Reed V. Teicoplanin therapy for Staphylococcus aureus septicaemia: relationship between pre-dose serum concentrations and outcome. J Antimicrob Chemother. 2000;45(6):835-41.

38. Kanazawa N, Matsumoto K, Ikawa K, Fukamizu T, Shigemi A, Yaji K, et al. An initial dosing method for teicoplanin based on the area under the serum concentration time curve required for MRSA eradication. J Infect Chemother. 2011;17(2):297-300.

39. Hagihara M, Umemura T, Kimura M, Mori T, Hasegawa T, Mikamo H. Exploration of optimal teicoplanin dosage based on pharmacokinetic parameters for the treatment of intensive care unit patients infected with methicillin-resistant Staphylococcus aureus. J Infect Chemother. 2012;18(1):10-6.

40. Matsumoto K, Watanabe E, Kanazawa N, Fukamizu T, Shigemi A, Yokoyama Y, et al. Pharmacokinetic/pharmacodynamic analysis of teicoplanin in patients with MRSA infections. Clin Pharmacol Adv Appl. 2016;8:15-8. 